\title{
Trascendencia de células hiperplásicas residuales a la prostatectomía radical. Revisión de la Literatura
}

\author{
Ramírez-Backhaus M, Trassierra M, Pontones JL, Luján S, DiCapua C, Rabenalt R*, \\ Stolzenburg JU*, Jiménez Cruz F. \\ Servicio de Urología. Hospital Universitario La Fe. Valencia. \\ *Departamento de Urología. Clínica Universitaria de Lepzig. Alemania
}

Actas Urol Esp. 2008;32(9):-873-878

\begin{abstract}
RESUMEN
TRASCENDENCIA DE CÉLULAS HIPERPLÁSICAS RESIDUALES A LA PROSTATECTOMÍA RADICAL. REVISIÓN DE LA LITERATURA

Introducción: La Prostatectomía radical es el tratamiento estándar para el cáncer de próstata organoconfinado. Clásicamente se presta atención a los márgenes positivos para células tumorales, pero no a la presencia de células hiperplásicas residuales a la cirugía capaces de generar antígeno prostático específico (PSA) y dificultar el seguimiento de los pacientes intervenidos. Esta situación nos lleva a plantear una revisión de la literatura, donde evaluemos la frecuencia de esta presencia, las posibles causas que lo justifiquen y la influencia de estas células en la evolución bioquímica de la enfermedad.

Material y Método: Realizamos una búsqueda bibliográfica a través de la base de datos "Pubmed" utilizando los términos "Mesh" "Prostatectomy" y "Prostatic Neoplasms" y "Prostate-Specific Antigen" a los que añadimos los términos "biochemical failure" y/o "hyperplasic cells" y/o "benign cells" . Asímismo, seleccionamos los trabajos en lengua inglesa, española y alemana, revisamos los artículos que dichos trabajos referencian e incluimos las series con más de 50 pacientes, cartas al editor, editoriales y revisiones de conjunto.

Conclusiones: La presencia de células hiperplásicas microscópicas residuales a la cirugía prostática radical, es un hecho frecuente que probablemente esté infra-evaluado. Los márgenes positivos para células benignas procederán del ápex o del cuello vesical donde la cápsula prostática no está bien definida, en ningún caso de la zona dorsolateral de la próstata, lo que traduciría un defecto de la técnica quirúrgica. La capacidad de estas células para generar PSA en cantidad suficiente para interferir en el seguimiento de los pacientes operados es controvertida. Recomendamos la inspección macroscópica de la pieza por el cirujano, tras la prostatectomía, para evaluar la integridad del ápex, zona craneal y cápsula dorsolateral.
\end{abstract}

Palabras clave: Cáncer de próstata. Prostatectomía radical. Células prostáticas hiperplásicas.

\section{ABSTRACT}

SIGNIFICANCE OF RESIDUAL HYPERPLASTIC CELLS AFTER RADICAL PROSTATECTOMY. A LITERATURE REVIEW

Introduction: Radical prostatectomy represents a standard surgical treatment for clinically localized prostate cancer. Classically pathologist and urologist worried about positive surgical margin, but not to the presence of surgery residual hyperplastic cells able to generate prostate specific antigen (PSA) and difficult the follow up of the patients that underwent surgery.

We reviewed the literature looking for the incidence, the potential etiology and the influence of these hyperplastic cells in the biochemical evolution of the disease.

Material and Method: The information for this review was compiled by searching the Pubmed database. We used "Mesh", Prostatectomy" and "Prostatic Neoplasms" and "Prostate-Specific Antigen" terms, and we added "biochemical failure" and/or "hyperplasic cells" and/or "benign cells". Furthermore, we select the work in English, Spanish and German, review articles that referenced this work and include the series with more than 50 patients, letters to the editor, editorials and overall reviews.

Conclusions: Benign hyperplasic cells left behind after radical prostatectomy are frequent and probably under-rated. The influence of those cells in the biochemical outcome is a controversial issue. Positive margins for benign cells can come from apex or neck of the bladder, where the prostatic capsule is not well defined, but no from dorso-lateral area,this would imply a technical mistake. We recommend the inspection of the specimen by the surgeon, after prostatectomy in order to detect apex integrity, cranial and dorso-lateral capsule. 
$\mathrm{E}^{1}$ objetivo de la cirugía oncológica exerética es la resección de todo el tejido tumoral. La cirugía radical de la glándula prostática con sus innumerables peculiaridades trasciende a esta máxima porque hasta el momento no es técnicamente factible la localización segura del tumor ni pre, ni intraoperatoriamente. Por otro lado el concepto de "prostatectomía con conservación de parénquima” dificultaría la monitorización posterior del paciente operado.

Desde que Walsh y Donker describieran con detalle la anatomía vasculonerviosa de la glándula prostática $^{1}$, los estudios sobre anatomía y técnica quirúrgica de la próstata, se han encaminado hacia la mejora de los resultados funcionales de la cirugía, pero siempre anteponiendo el control oncológico de la enfermedad.

La continencia es la variable funcional de mayor influencia en la calidad de vida de los pacientes intervenidos. La disección roma de la uretra adyacente al ápex prostático para alargar el muñón uretral distal y por ende preservan el esfinter externo está relacionada con la mejora de la continencia postoperatoria ${ }^{2,3}$.

Por otro lado la disección cuidadosa del cuello vesical aumenta la probabilidad de continencia inmediata tras la retirada del catéter, si bien, las ventajas de esta técnica en la tasa global de continencia tardía es controvertida ${ }^{4,5}$.

La preservación de las bandeletas vasculonerviosas, llega a su máxima expresión con el desarrollo de la cirugía mínimamente invasiva, la laparoscopia y cirugía asistida por robot.

El urólogo debe ser consciente de que en todo momento priman los resultados oncológicos sobre los funcionales por lo que la aplicabilidad de estas técnicas dependerá de los factores pronósticos de recidiva: valor del PSA pre operatorio, grado de Gleason, tacto rectal, ecografía transrectal, número y localización de los cilindros positivos. Pero estas modificaciones quirúrgicas que, sin duda, mejoran los resultados funcionales, teóricamente podrían aumentar, independientemente del estadio clínico, el riesgo de márgenes quirúrgicos positivos.

Generalmente cuando evaluamos los márgenes quirúrgicos, los propios patólogos y los urólogos atendemos a la presencia de células tumorales que contactan con el borde de la pieza quirúrgica, generalmente marcado con tinta china. Olvidando, en cierta medida, informar o valorar roturas durante la disección de la cápsula prostática que podrían traducir la presencia de células hiperplásicas benignas en el lecho quirúrgico. Más complicado es, si cabe, el análisis histológico en el ápex y en la porción más craneal de la próstata, donde la ausencia de cápsula prostática, hace difícil precisar si la exéresis ha sido completa ${ }^{14}$. Para descubrir la presencia de células hiperplásicas residuales en dichas zonas sería necesario realizar biopsias intraoperatorias del rodete uretral distal y del cuello vesical, o biopsias de la anastomosis.

El principal recurso del que disponemos para el seguimiento de los pacientes intervenidos, es la determinación del nivel sérico del PSA. Varios grupos, ante pacientes con PSA en rangos de recidiva bioquímica, en ausencia de signos de enfermedad diseminada o recidiva local, se han planteado la posibilidad de que células hiperplásicas residuales sean las responsables del aumento del nivel sérico de PSA poscirugía en un paciente con estadio patológico pT2, por ejemplo.

\section{MATERIAL Y MÉTODO}

Realizamos una búsqueda bibliográfica a través de la base de datos "Pubmed" utilizando los términos "Mesh" "Prostatectomy" y "Prostatic Neoplasms" y "Prostate-Specific Antigen" a los que añadimos los términos "biochemical failure" y/o "hyperplasic cells" y/o "benign cells". Así mismo, seleccionamos los trabajos en lengua inglesa, española y alemana, revisamos los artículos que dichos trabajos refieren e incluimos las series con más de 50 pacientes, cartas al editor, editoriales y revisiones de conjunto.

\section{RESULTADOS Y DISCUSIÓN}

En la Tabla 1 exponemos las series de casos incluidas en esta revisión; se trata de estudios en los que mediante la toma de biopsias intraoperatorias evalúan la presencia de células hiperplásicas residuales en el lecho quirúrgico; describimos las características clínicas y patológicas de la muestra estudiada en cada trabajo y la metodología técnica.

Como hemos indicado previamente, las zonas donde técnicamente es más probable la presencia de células hiperplásicas tras la exéresis de la próstata son el ápex y el cuello vesical y las zonas dorsolaterales.

El esfinter externo asegura la continencia tras la cirugía prostática. A nivel del ápex prostático fibras 
Tabla 1. Trabajos que evaluan los márgenes quirúrgicos para células prostáticas benignas tras la prostatectomía radical.

\begin{tabular}{|c|c|c|c|c|c|c|c|c|c|}
\hline \multirow[b]{2}{*}{ Ref. } & \multirow[b]{2}{*}{$\mathbf{n}$} & \multicolumn{2}{|c|}{ E. Clínico } & \multicolumn{2}{|c|}{ E. Patológico } & \multirow{2}{*}{$\begin{array}{c}\text { Margenes + } \\
\%\end{array}$} & \multirow[t]{2}{*}{ Localizacion Bx Intraop } & \multirow{2}{*}{$\begin{array}{c}\text { Bx+ Tumor } \\
\%\end{array}$} & \multirow{2}{*}{$\begin{array}{c}\mathrm{Bx}+\mathrm{HBP} \\
\%\end{array}$} \\
\hline & & $\begin{array}{l}\text { cT1c } \\
\%\end{array}$ & $\begin{array}{c}\text { cT2 } \\
\%\end{array}$ & $\begin{array}{c}\text { pT2 } \\
\%\end{array}$ & $\begin{array}{l}\text { pT3 } \\
\%\end{array}$ & & & & \\
\hline Shah et al. ${ }^{16}$ & 95 & 81 & 18 & 65 & 35 & 17 & Rodete uretral adyacente al apex & 4 & 41 \\
\hline Wood et al. ${ }^{33}$ & 73 & nd & nd & 44 & 56 & 26 & cuello & 12 & 7 \\
\hline Lepor y Kaci ${ }^{21}$ & 500 & 85 & 15 & nd & nd & nd & $\begin{array}{l}\text { rodete uretral adyacente apex } \\
\text { cuello }\end{array}$ & $\begin{array}{l}4,5 \\
0,8\end{array}$ & $\begin{array}{l}34,6 \\
32,7\end{array}$ \\
\hline Lepor et al. ${ }^{17}$ & 100 & 70 & 30 & 75 & 25 & nd & cuello & $3 \%$ & $57 \%$ \\
\hline Djavan et al. ${ }^{18}$ & 351 & nd & nd & nd & nd & nd & márgenes pieza de prostatectomia & 8 & 27 \\
\hline Paul et al. ${ }^{22}$ & 802 & nd & nd & 75 & 25 & 19,55 & Rodete uretral adyacente al apex & & 73,6 \\
\hline Dillenburg et al. ${ }^{19}$ & 198 & 18 & 82 & 69 & 30 & 13 & $\begin{array}{l}\text { apex } \\
\text { cuello }\end{array}$ & $\begin{array}{c}5 \\
0,5\end{array}$ & $\begin{array}{l}16 \\
5,5\end{array}$ \\
\hline Eichelberg $^{20}$ & 604 & 70,4 & 29,4 & 79 & 20,23 & 14,47 & idem & 0,5 & 5,5 \\
\hline
\end{tabular}

musculares estriadas de dicho músculo se solapan con la superficie prostática especialmente a nivel ventral. En el ápex, la próstata está en íntimo contacto no sólo con dicho esfínter sino con estructuras como los ligamentos puboprostáticos y la fascia endopélvica, incluso en ocasiones con el elevador del ano. La tendencia actual es a practicar una disección lo más atraumática posible, con el objetivo de conseguir un muñón uretral largo que asegure la continencia postoperatoria. Indudablemente, todas estas circunstancias aumentan la probabilidad de dejar células hiperplásicas residuales.

La próstata en su región posterior carece de cápsula y en ocasiones su separación de la vejiga, muy próxima al borde del esfínter interno, se verá dificultada especialmente cuando el cirujano pretenda conservar el cuello vesical o esfínter interno. Si el paciente desarrolla adenomas intravesicales o un lóbulo medio, la conservación del cuello es prácticamente imposible. Ante cualquiera de las anteriores circunstancias es factible la presencia de focos de células hiperplásicas a nivel del cuello, pero en ningún caso se debe permitir la persistencia de un adenómero completo.

Durante la prostatectomía radical el cirujano es conocedor de la presencia de fibras nerviosas que discurren en íntimo contacto a la superficie prostática a nivel dorsal, lateral y posterior; lo que conocemos como bandeletas o haces neurovasculares. Este término tan extendido, en realidad no tiene una correlación anatómica exacta pero se utiliza para describir los pedículos vasculares dorsolaterales.
A dicho nivel, especialmente cuando se llevan a cabo disecciones intrafasciales, es factible la disrupción de la cápsula y la persistencia de nidos celulares hiperplásicos en el lecho quirúrgico. Pero la presencia de focos de células benignas traduce una técnica quirúrgica defectuosa, pues la cápsula es el límite quirúrgico que en ningún caso se debe sobrepasar.

La metodología utilizada por los diferentes grupos para la detección de restos hiperplásicos ha sido: biopsias transrectales de la anastomosis ${ }^{15}$, biopsias intraoperatorias del ápex ${ }^{16}$, del cuello vesical $^{17}$ de la propia próstata ${ }^{18}$ o, cuando existía sospecha de rotura capsular, de la bandeleta adyacente $^{19}$.

La tasa de tejido adenomatoso residual en el cuello vesical oscila, según las series, entre el 5,5\% ${ }^{20}$ y el $32,7^{21}$ y en el ápex entre el $16 \%{ }^{19}$ y el $73 \%^{22}$. Según Dilenburg et al. la disección en la técnica laparoscópica es más minuciosa y las mejoras en la visión justifican que en su serie la tasa sea la más baja $^{19}$.

Djavan et al. en una serie de 351 prostatectomías radicales, refiere un $27 \%$ de pacientes que presentaban márgenes positivos para glándulas hiperplásicas ${ }^{18}$ Cuando estratificaron sus resultados en función del estadio patológico, objetivaron que el $79 \%$ de los pacientes pT2 con márgenes positivos para estructuras glandulares benignas expresaban PSA en el seguimiento a largo plazo. Djavan et al. consideraron que, en la mayoría de los casos, dicho PSA sería producido por el componente epitelial 
residual y no por células tumorales puesto que los márgenes para adenocarcinoma eran negativos ${ }^{18}$

Por un lado parece un hecho contrastado que tras la prostatectomía radical, con una frecuencia variable, persisten células hiperplásicas, pero en la literatura objetivamos cierto debate entre Djavan ${ }^{23} \mathrm{y}$ el resto de los grupos, respecto a la trascendencia de dichas células y su potencial capacidad de sintetizar PSA.

Los datos de Djavan presentan una peculiaridad: en la mayoría de los casos los márgenes eran positivos para células benignas en la zona prostática adyacente a las bandeletas. Dicho hallazgo a nuestro juicio es inadmisible pues, a diferencia del ápex o del cuello vesical, y aunque se lleve a cabo una cirugía conservadora de bandeletas vasculonerviosas en ningún caso está justificada la apertura o disección intracapsular, ya que la mayoría de tumores se generan en la zona periférica y el riesgo de márgenes positivos para adenocarcinoma sería muy alto.

Djavan et al. proponen que el punto de corte para la recidiva bioquímica debería elevarse para los pacientes pT2 con márgenes positivos para células benignas $^{23}$ sobre la base de un trabajo donde el $61 \%$ de los pacientes con dichas características superaba $0,2 \mathrm{ng} / \mathrm{ml}^{18}$. Además describieron una dinámica bioquímica característica en su muestra; los pacientes alcanzaban un nadir de PSA $<0,1$ en 7 semanas y padecían un progresivo y lento incremento en los valores séricos hasta alcanzar una meseta de $0,9 \mathrm{ng} / \mathrm{ml}^{18}$, aproximadamente al año de la cirugía. Sin embargo, otros trabajos también evaluaron la evolución del PSA de los pacientes en los que se objetivaron células hiperplásicas en alguna de las biopsias intraoperatorias y no observaron los hallazgos descritos por Djavan. Tampoco el trabajo de Dillenburg, con un seguimiento medio de 8 meses, parece capaz de resolver las dudas ${ }^{18}$. Sin embargo, en un trabajo previo, con mayor casuística y con un seguimiento medio de 48 meses, Paul et al. no observaron la dinámica bioquímica referida ${ }^{22}$, ni siquiera cuando estratificaron sus resultados según la cantidad de tejido adenomatoso en las biopsias ${ }^{22}$.

Por otro lado Boccon-Gibon et al. ${ }^{24}$ en un trabajo donde comparaban el riesgo de disrupción de la cápsula prostática durante la cirugía retropúbica con la perineal, evaluaron la presencia de márgenes positivos para adenocarcinoma y para células benignas y la evolución bioquímica de ambos grupos.
Observaron que las recidivas bioquímicas se daban de forma significativamente más frecuente en el primer grupo $^{24}$.

Raveri et al. ante un grupo de pacientes en estadio pT2 y progresión bioquímica, re-evaluaron la histología realizando cortes cada $2 \mathrm{~mm}$, buscando cualquier disrupción de la cápsula prostática que justificara la presencia de remananentes glandulares benignos; sin embargo, los hallazgos se inclinaban en mayor medida hacia una recidiva local o metastásica, cuando no objetivaban hallazgos histológicos de interés ${ }^{25}$.

Por tanto, nos parece que las reflexiones de Djavan $^{23}$ son inconsistentes, y las disertaciones que hace al respecto de la potencial capacidad de malignizar de estos restos benignos, así como la proposición de iniciar un tratamiento profiláctico con inhibidores de la 5 alfa-reductasa en estos pacientes, carece de base científica ${ }^{23}$.

La dinámica del PSA, el cociente PSA libre/total, tanto en suero como en orina, y sobre todo las biopsias de la anastomosis son algunas de las variables que podrian resolver las dudas sembradas por estos grupo $^{26}$.

Un crecimiento exponencial en los valores del PSA después de un nadir $<0,1 \mathrm{ng} / \mathrm{ml}$ es compatible con una recurrencia, sin embargo un PSA persistente con un crecimiento lento y/o un tiempo de duplicación largo indicarían la presencia de células hiperplásicas tras la cirugía. Djavan et al. consideran que fenómenos como la angiogénesis de las células residuales, y los procesos inflamatorios justifican un PSA nadir inferior a $0,1 \mathrm{ng} / \mathrm{ml}$ en los pacientes con márgenes positivos para células hiperplásicas ${ }^{24}$.

El tiempo de duplicación del PSA, parece un arma útil en la evaluación de estos pacientes. Según el trabajo de Roberts y cols. Cuando un paciente tiene un tiempo de duplicación de 10 años, la supervivencia libre de recidiva local o progresión sistémica es del 87\%, del 62\% cuando el tiempo de duplicación está entre 1 y 10 años y del 46\% cuando el PSA duplica su valor entre 1y 6 meses. Si aplicamos estos datos a los pacientes de la serie de Djavan et al. (pacientes con estadio pT2 que alcanzan un nadir de 0,1 tienen un PSA medio de $0,9 \mathrm{ng} / \mathrm{ml}(0,2-$ 2) a los cinco años), intuimos que probablemente en muchos de ellos la elevación del PSA se deba a una progresión o recidiva. 
Algunos grupos intentaron evaluar, sin éxito, si la medición del PSA en orina podría ayudar al diagnóstico de una recidiva local ${ }^{27}$. Probablemente el fracaso de dichos trabajos residía en que el epitelio de las glándulas paruretrales también sintetiza esta proteína $^{28}$. Posteriormente se planteo la posibilidad de medir el PSA total y la fracción libre del PSA en orina antes y tras el masaje de la anastomosis ${ }^{27}$. Los primeros resultados parecían prometedores, la medición de la fracción libre de PSA en el chorro miccional medio permitiría distinguir una recidiva tumoral local de una recidiva hiperplásica. No existen datos posteriores, lo que parece indicar que del mismo modo que las mediciones de la fracción libre del PSA en suero no son útiles, tampoco lo van a ser las mediciones en orina ${ }^{29}$.

La biopsia transrectal es un procedimiento invasivo utilizado por algunos grupos para detectar una recidiva local. Los estudios asocian valores elevados de PSA $>1 \mathrm{ng} / \mathrm{ml}$ con biopsias positivas $^{30}$ y con el estadio patológico ${ }^{15}$, pero muy pocos evalúan o ni siquiera mencionan la presencia de células hiperplásicas en estas biopsias, no sabemos si en realidad no las había o si los clínicos no le otorgaban la relevancia pertinente ${ }^{15,31}$.

Fowler et al. realizaron biopsia transrectal de la anastomosis a 62 pacientes con recidiva bioquími$\mathrm{ca}^{32}$. En un $10 \%$ las biopsias revelaban la presencia, única de células prostáticas hiperplásicas, no obstante en dicho 10\% la evolución del PSA fue exponencial y sugería claramente la coexistencia de células tumorales con las hiperplásicas detectadas. La biopsia transrectal de la anastomosis no es, a nuestro juicio, suficientemente sensible ni específica para ser aplicada en los pacientes que nos ocupan.

\section{CONCLUSIONES}

La presencia de células hiperplásicas residuales a la cirugía prostática radical, parece un hecho relativamente frecuente probablemente infra-evaluado. Las zonas afectas más frecuentes son: el ápex, por el esfuerzo de los cirujanos en la preservación del esfínter externo, y el cuello vesical por la impronta del adenoma en el mismo. La presencia de márgenes positivos para células hiperplásicas en la zona dorsal que se corresponde con la zona periférica es a nuestro juicio, reflejo de una práctica quirúrgica subóptima, por el riesgo de asociar márgenes quirúrgicos para células tumorales.
La viabilidad de dichas células es un hecho contrastado, en cuanto a que se han objetivado células de este tipo en biopsias de la anastomosis.

Su capacidad para generar PSA en cantidad suficiente para interferir en el seguimiento de los pacientes operados es controvertida y ningún grupo lo ha podido confirmar hasta la fecha.

Consideramos recordable la toma de imágenes de la próstata durante la ecografía transrectal de la biopsia prostática, o bien la práctica de cistoscopia previa a la intervención; para conocer la morfología de la glándula prostática, y evitar la presencia residual de un lóbulo medio.

A nuestro juicio no está justificada la toma de dichas biopsias con tal fin ni las biopsias transrectales de la anastomosis por la escasa especificidad.

\section{REFERENCIAS}

1. Walsh PC, Donker PJ. Impotence following radical prostatectomy: insight into etiology and prevention. The Journal of urology. 1982 Sep;128(3):492-497.

2. McGuire E. Urethral sphincter mechanisms. The Urologic clinics of North America. 1979 Feb;6(1):39-49.

3. Myers RP, Goellner JR, Cahill DR. Prostate shape, external striated urethral sphincter and radical prostatectomy: the apical dissection. The Journal of urology. 1987 Sep;138(3):543-550.

4. Lowe BA. Comparison of bladder neck preservation to bladder neck resection in maintaining postrostatectomy urinary continence. Urology. 1996 Dec;48(6):889-893.

5. Licht MR, Klein EA, Tuason L, Levin H. Impact of bladder neck preservation during radical prostatectomy on continence and cancer control. Urology. 1994 Dec;44(6):883-887.

6. Tewari A, Takenaka A, Mtui E, Horninger W, Peschel R, Bartsch G, et al. The proximal neurovascular plate and the trizonal neural architecture around the prostate gland: importance in the athermal robotic technique of nerve-sparing prostatectomy. BJU international. 2006 Aug;98(2):314-323.

7. Takenaka A, Leung RA, Fujisawa M, Tewari AK. Anatomy of autonomic nerve component in the male pelvis: the new concept from a perspective for robotic nerve sparing radical prostatectomy. World journal of urology. 2006 Jun;24(2): 136-143.

8. Kessler TM, Burkhard FC, Studer UE. Nerve-sparing open radical retropubic prostatectomy. European urology. 2007 Jan;51(1):90-97.

9. Curto F, Benijts J, Pansadoro A, Barmoshe S, Hoepffner JL, Mugnier C, et al. Nerve sparing laparoscopic radical prostatectomy: our technique. European urology. 2006 Feb;49(2):344-352.

10. Stolzenburg JU, Rabenalt R, Do M, Schwalenberg T, Winkler M, Dietel A, et al. Intrafascial Nerve-Sparing Endoscopic Extraperitoneal Radical Prostatectomy. European urology. 2007 Dec 3.

11. Rassweiler J. Intrafascial nerve-sparing laproscopic radical prostatectomy: do we really preserve relevant nerve-fibres? European urology. 2006 Jun;49(6):955-957.

12. Kaul S, Savera A, Badani K, Fumo M, Bhandari A, Menon M. Functional outcomes and oncological efficacy of Vattikuti Institute prostatectomy with Veil of Aphrodite nerve-sparing: an analysis of 154 consecutive patients. BJU international. 2006 Mar;97(3):467-472. 
13. Albers P, Schafers S, Lohmer H, de Geeter P. Seminal vesiclesparing perineal radical prostatectomy improves early functional results in patients with low-risk prostate cancer. BJU international. 2007 Nov;100(5):1050-1054.

14. Stamey TA, Villers AA, McNeal JE, Link PC, Freiha FS. Positive surgical margins at radical prostatectomy: importance of the apical dissection. The Journal of urology. 1990 Jun; 143(6): 1166-72; discussion 72-73.

15. Shekarriz B, Upadhyay J, Wood DP, Jr., Hinman J, Raasch J, Cummings GD, et al. Vesicourethral anastomosis biopsy after radical prostatectomy: predictive value of prostate-specific antigen and pathologic stage. Urology. 1999 Dec;54(6):10441048.

16. Shah O, Melamed J, Lepor H. Analysis of apical soft tissue margins during radical retropubic prostatectomy. The Journal of urology. 2001 Jun;165(6 Pt 1):1943-8; discussion 8-9.

17. Lepor H, Chan S, Melamed J. The role of bladder neck biopsy in men undergoing radical retropubic prostatectomy with preservation of the bladder neck. The Journal of urology. 1998 Dec;160(6 Pt 2):2435-2439.

18. Djavan B SI, Hruby S, Susani M, Haitel A, Eremad M, Mostofi MK, McLeos D, Marberger M. Benign prostatic glands in the surgical margin of radical prostatectomies: redefining PSA nadir. AUA Congress: Journal of Urology 2000.

19. Dillenburg W, Poulakis V, Witzsch U, de Vries R, Skriapas K, Altmansberger HM, et al. Laparoscopic radical prostatectomy: the value of intraoperative frozen sections. European urology. 2005 Oct;48(4):614-621.

20. Eichelberg C, Erbersdobler A, Haese A, Schlomm T, Chun FK, Currlin E, et al. Frozen section for the management of intraoperatively detected palpable tumor lesions during nerve-sparing scheduled radical prostatectomy. European urology. 2006 Jun;49(6):1011-6; discussion 6-8.

21. Lepor H, Kaci L. Role of intraoperative biopsies during radical retropubic prostatectomy. Urology. 2004 Mar;63(3):499-502.

22. Paul R, Hoppmann M, van Randenborgh $H$, Kubler $H$, Alschibaja M, Gunther M, et al. Residual benign prostatic glands at the urethrovesical anastomosis after radical retropubic prostatectomy: prediction and impact on disease outcome. European urology. 2004 Sep;46(3):321-326.

23. Djavan B, Milani S, Fong YK. Benign positive margins after radical prostatectomy means a poor prognosis-pro. Urology. 2005 Feb;65(2):218-220.

24. Boccon-Gibod L, Ravery V, Vordos D, Toublanc M, Delmas V, Boccon-Gibod L. Radical prostatectomy for prostate cancer: the perineal approach increases the risk of surgically induced positive margins and capsular incisions. The Journal of urology. 1998 Oct; $160(4): 1383-1385$.
25. Ravery V, De la Taille A, Toublanc M, Boccon-Gibod L, Hermieu JF, Delmas V, et al. Prostate specimen reevaluation in patients with organ confined prostate cancer and postoperative biological recurrence. The Journal of urology. 1996 Jun;155(6):1981-1982.

26. Ravery V. The significance of recurrent PSA after radical prostatectomy: benign versus malignant sources. Seminars in urologic oncology. 1999 Aug;17(3):127-129.

27. Malavaud B, Salama G, Miedouge M, Vincent C, Rischmann P, Sarramon JP, et al. Influence of digital rectal massage on urinary prostate-specific antigen: interest for the detection of local recurrence after radical prostatectomy. The Prostate. 1998 Jan $1 ; 34(1): 23-28$

28. Iwakiri J, Granbois K, Wehner N, Graves HC, Stamey T. An analysis of urinary prostate specific antigen before and after radical prostatectomy: evidence for secretion of prostate specific antigen by the periurethral glands. The Journal of urology. 1993 Apr; 149(4):783-786.

29. Wojno KJ, Vashi AR, Schellhammer PF, Wright GL, Jr., Montie JE. Percent free prostate-specific antigen values in men with recurrent prostate cancer after radical prostatectomy. Urology. 1998 Sep;52(3):474-478.

30. Scattoni V, Roscigno M, Raber M, Montorsi F, Da Pozzo L, Guazzoni G, et al. Multiple vesico-urethral biopsies following radical prostatectomy: the predictive roles of TRUS, DRE, PSA and the pathological stage. European urology. 2003 Oct;44(4): 407-414.

31. Deliveliotis C, Manousakas T, Chrisofos M, Skolarikos A, Delis A, Dimopoulos C. Diagnostic efficacy of transrectal ultrasoundguided biopsy of the prostatic fossa in patients with rising PSA following radical prostatectomy. World journal of urology. 2007 Jun;25(3):309-313.

32. Fowler JE, Jr., Brooks J, Pandey P, Seaver LE. Variable histology of anastomotic biopsies with detectable prostate specific antigen after radical prostatectomy. The Journal of urology. 1995 Mar;153(3 Pt 2):1011-1014.

33. Wood DP, Jr., Peretsman SJ, Seay TM. Incidence of benign and malignant prostate tissue in biopsies of the bladder neck after a radical prostatectomy. The Journal of urology. 1995 Oct; 154 (4): 1443-1446.

Correspondencia autor: Dr. M. Ramírez-Backhaus Servicio de Urología. Hospital Universitario La Fe Avda. Campanar, 21 - 46009 Valencia

Tel.: 963862700

E-mail autor: ramirezbackhaus@yahoo.es

Información artículo: Original - Cáncer de próstata

Trabajo recibido: mayo 2008

Trabajo aceptado: julio 2008 